\title{
Upregulated Expression of Actin-Like 6A is a Risk Factor Affecting the Prognosis of Pancreatic Cancer
}

\section{Zhong Zhang \\ Haochun Guo \\ Haijun Zhang (D)}

Department of Oncology, Zhongda Hospital, Medical School of Southeast University, Nanjing, Jiangsu, People's Republic of China
Correspondence: Haijun Zhang Department of Oncology, Zhongda Hospital, Medical School of Southeast University, 87 Dingjiaqiao Road, Nanjing, Jiangsu, 210009, People's Republic of China

Tel/Fax +862583275416

Email zhanghaijunseu@।63.com
Purpose: Actin-like 6A (ACTL6A), a regulatory subunit of the ATP-dependent chromatinremodeling complex SWI/SNF, acts as an oncogenic factor. This study is aimed at evaluating the correlation between ACTL6A expression and clinicopathological parameters in pancreatic cancer (PC) patients.

Methods: The differences of Actl6a mRNA expression between PC tissues and normal pancreatic tissues were analyzed in public databases, and ACTL6A expression was then determined and confirmed in 60 paired tissue specimens using immunohistochemistry staining. The association analysis between ACTL6A expression and the clinicopathological characteristics was analyzed, as well as Kaplan-Meier survival analysis. Univariate and multivariate Cox analyses were performed to identify the prognostic factors in the overall survival (OS) of patients with PC.

Results: The mRNA expression of Actl6a showed significantly higher in PC compared to normal controls $(p<0.05)$ from public databases. The score of immunohistochemistry staining further confirmed that ACTL6A expression was significantly upregulated in PC tissues $(\mathrm{p}<0.001)$ through immunohistochemistry staining. High ACTL6A expression was associated with lymphovascular space invasion of PC. Kaplan-Meier analysis revealed that the high expression of ACTL6A was markedly associated with poor OS. Moreover, univariate and multivariate analysis demonstrated that ACTL6A acted as an independent risk factor for PC prognosis.

Conclusion: ACTL6A is upregulated in PC and acts as a risk factor for poor prognosis in patients with $\mathrm{PC}$, and therefore clinicians could around it design preventive measures and individualized treatment to improve mortality in patients with PC.

Keywords: pancreatic cancer, risk factors, actin-like 6A, prognosis, biomarkers

\section{Introduction}

Pancreatic cancer (PC) with high aggressiveness and malignancy has become an enormously common cancer of the digestive system during 10 years. Globally, the 5-year overall survival (OS) rate of patients with PC is less than $9 \%$, and the mortality rate is predicted to peak by $2030 .{ }^{1}$ Due to insidious symptoms, only less than $10 \%$ of PC is initially diagnosed with a local stage, and the prognosis of PC is extremely poor. ${ }^{2}$ Therefore, further investigation into novel cancer-related genes is required and meaningful for the improvement of prognosis.

SWI/SNF complexes are evolutionarily conserved multi-subunit molecular machines that mediate transcriptional regulation ${ }^{3}$ and are linked to a poor prognosis across several cancer types. ${ }^{4-6}$ Among them, Actin-like 6A (ACTL6A), encoded by Actl6a, acts as a chromatin-remodeling factor and regulates the function of 
progenitor and stem cell transcriptionally. ${ }^{7,8}$ In addition, ACTL6A expression is associated with prognosis in many types of cancer, such as hepatocellular carcinoma, ${ }^{9}$ colon cancer, ${ }^{10}$ and esophageal squamous cell carcinoma. ${ }^{11}$ Recently, research revealed that epithelial-tomesenchymal transition (EMT) was also regulated by ACTL6A. ${ }^{9,12}$ In addition, the study showed that ACTL6A overexpression could lead to increased repair of cisplatin-DNA adducts and cisplatin resistance. ${ }^{13}$ However, the role of ACTL6A in tumorigenicity and clinical prognosis of $\mathrm{PC}$ remains unclear so far.

In this connection, we analyzed the differences of ACTL6A expression in PC tissues and normal tissues, and we investigated the prognostic effect of ACTL6A on $\mathrm{PC}$ based on cases in public databases and confirmed it in our center.

\section{Materials and Methods}

\section{Analysis of Database}

Differential expression of Actl6a mRNA between pancreatic tumor and normal tissues was analyzed using the Gene Expression Profiling Interactive Analysis website (GEPIA; http://gepia.cancer-pku.cn/). Data for 179 patients with PC and 171 normal tissue samples analyzed on the GEPIA website were obtained from TCGA and normal tissue samples from Genotype-Tissue Expression (GTEx). ${ }^{14-16}$ The gene expression, determined as transcripts per million (TPM), was calculated by $\log 2(\mathrm{TPM}+1)$ for comparison. Based on the expression levels of Actl6a mRNA, the overall survival (OS) of patients was also analyzed.

\section{Patients and Tissue Specimens}

A total of 60 patients with PC confirmed by histopathology from January 2013 to June 2020 at Zhongda Hospital, Medical School of Southeast University were selected for the study. Sixty paired pancreatic tumor and normal tissues from patients who did not receive chemotherapy or radiotherapy were obtained to detect ACTL6A expression. Any patients with incomplete epidemiological and clinical information or lack of follow-up information were excluded. The results of serum tumor markers were collected from 60 healthy individuals who were admitted to the hospital for physical examination at the same time. All patients provided informed consent. Patients were followed up by telephone or at office visits every 3 months from the end date of surgery. The latest follow-up ended in July 2021. According to the eighth edition of the American
Joint Committee on Cancer (AJCC) Cancer Staging Manual, pathological stages were validated. The study was conducted with approval from the ethics committee of Zhongda Hospital, Southeast University. The study protocol protected the private information of enrolled patients in accordance with the provisions of the Helsinki Declaration.

\section{Immunohistochemistry Staining}

The paraffin-embedded pathological specimens were cut into $4-\mu \mathrm{m}$-thick sections. After being dewaxed in xylene and rehydrated in grade alcohol, the paraffin sections were submerged in a pH 6.0 citric acid solution and heated at $95^{\circ} \mathrm{C}$ for approximately 15 minutes for antigen retrieval. Next, the sections were incubated with rabbit ACTL6A antibody (Abcam Corp, USA, diluted 1:200) overnight at $4^{\circ} \mathrm{C}$ and washed 3 times with phosphate buffer saline (PBS). The sections were then incubated with horseradish peroxidase-conjugated secondary antibody for 30 minutes at room temperature in the dark. After stained with freshly prepared 3,3'-diaminobenzidine (DAB), they were counterstained with hematoxylin and differentiated with $1 \%$ hydrochloric acid. PBS was used to substitute the primary antibody as negative control. Finally, the sections were dehydrated with alcohol and sealed with neutral gum, and pictures were taken by microscope for positive cell calculation. Immunohistochemical staining analysis was performed independently by two pathologists according to the staining intensity and the percentage of positive cells. The staining intensities were 0 (negative), 1 (positive 1+), 2 (positive $2+$ ), and 3 (positive $3+$ ), respectively. The percentages of cells were 0 (negative), 1 (1-25\%), 2 (26-50\%), 3 (51-75\%), and 4 (76-100\%), respectively. ${ }^{17,18}$ Total scores were calculated by multiplying the scores of staining intensity and percentage.

\section{Statistical Analysis}

Statistical analyses and mapping were performed using SPSS software (version 18.0, IBM Corporation, Armonk, NY, USA), GraphPad Prism (Version 8.4.3, GraphPad Software, La Jolla, CA, USA), and R (version 3.4.1, http://www.r-pro ject.org/) in the present study. Wilcoxon test was used to evaluate significant differences between pancreatic cancer and normal tissues, and the $\chi 2$ test and continuity correction were used to explore the relationship between ACTL6A expression and clinicopathological features. The diagnostic efficiency of ACTL6A expression was analyzed through receiver operating characteristic (ROC) curves for PC. The 
sensitivity and specificity were evaluated at an optimal cutoff. The expression of ACTL6A was classified as high expression and low expression according to the cutoff. Survival analysis was analyzed using Kaplan-Meier curve, and difference among groups was assessed using Log rank test. Both univariable and multivariable analyses were used in survival analysis, respectively. The clinicopathological factors with significant associations $(\mathrm{p}<0.1)$ in the aforementioned univariable analysis were subjected to multivariate analysis. $\mathrm{p}<0.05$ was considered to be statistically significant.

\section{Results}

\section{ACTL6A Expression in PC and Normal Tissues}

To explore the potential role of ACTL6A in PC, the expression of Actl6a mRNA was analyzed with the publicly available
GEPIA database. In clinical PC specimens $(n=178)$ and normal tissues $(\mathrm{n}=171)$, Actl6a mRNA had significant differential expression between the two groups. What is more, Actl6a mRNA was upregulated in PC than normal tissues ( $p$ $<0.05$, Figure 1A). Then, the protein expression of ACTL6A was validated and compared in PC samples $(n=60)$ and normal tissues $(n=60)$ with immunohistochemistry staining in our center. The typical immunohistochemical results of normal tissues and PC tissues are shown in Figure 1B, which demonstrated that ACTL6A was mainly observed in the nucleus of cells. By multiplying the staining intensity and percentage, the protein expression of ACTL6A was also overexpressed in pancreatic cancer $(\mathrm{p}<0.001$, Figure $1 \mathrm{C})$. Table 1 shows the number of patients with different scores based on immunohistochemistry staining. The results above indicated that ACTL6A was upregulated in PC.
A
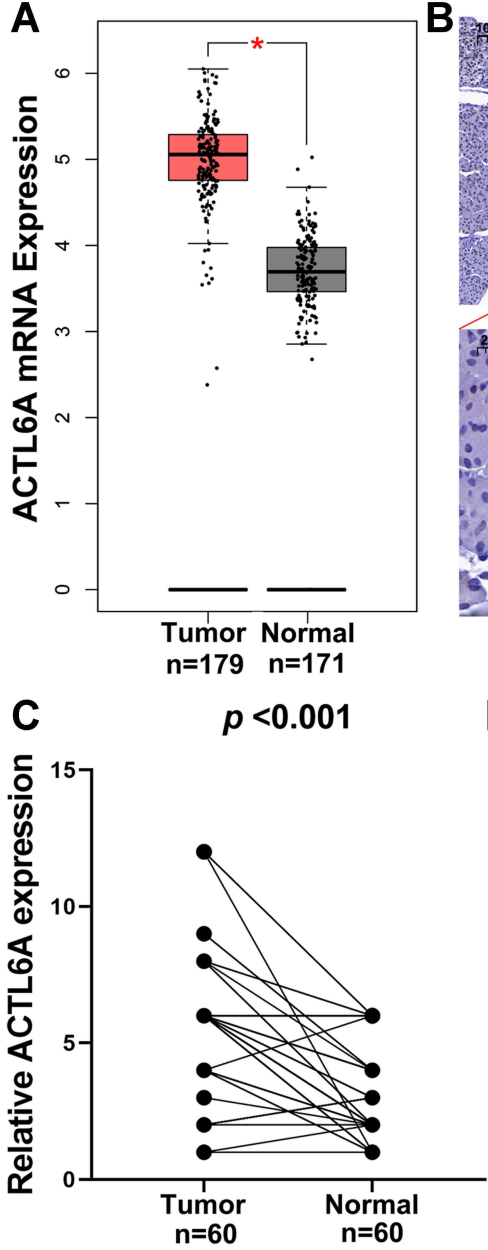

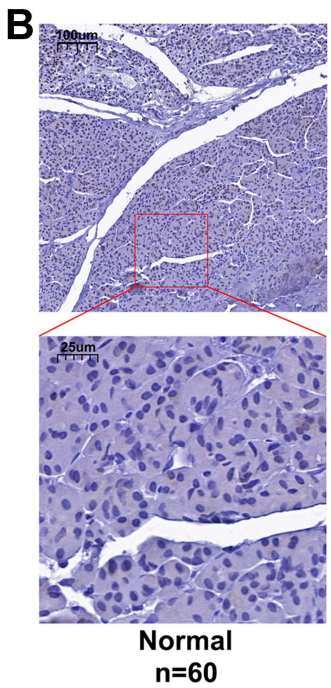

D 0

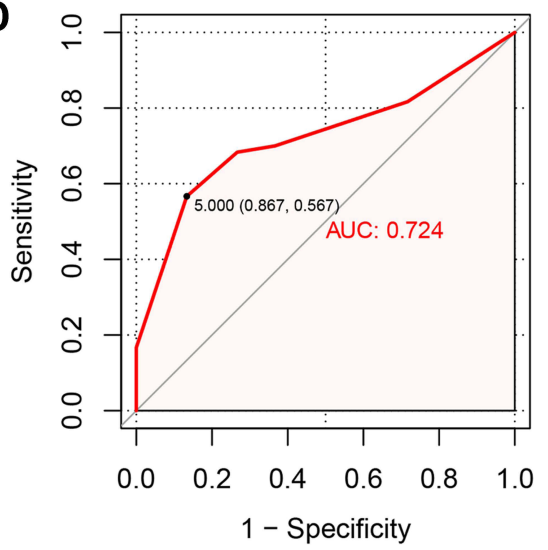

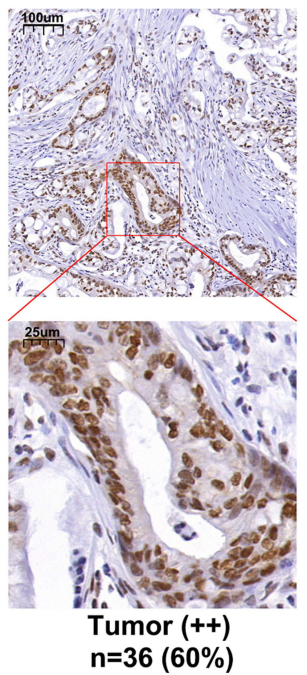
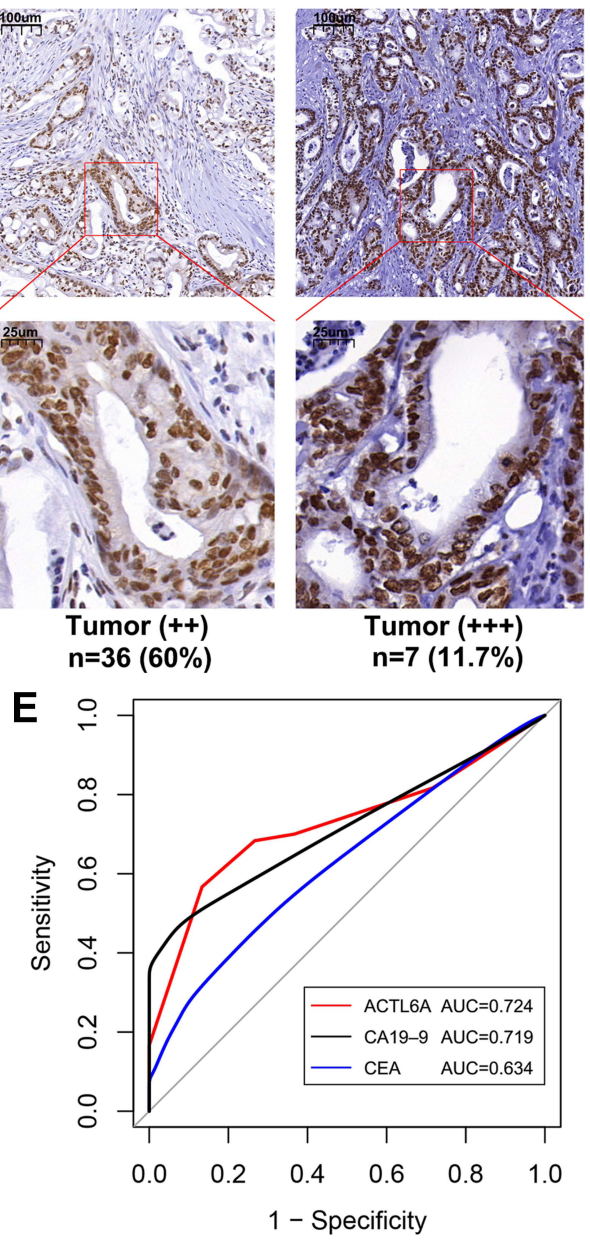

Figure I Expression of ACTL6A in PC and normal tissues. (A) Differential expression of Actl6a mRNA between pancreatic tumor and normal tissues. (B) Immunohistochemical results of typical normal tissues and PC tissues with different staining intensities. (C) Differential expression of ACTL6A between pancreatic tumor and normal tissues. (D) ACTL6A represented a moderate diagnostic value. The ROC of pancreatic cancer samples and normal tissues. (E) ROC for the diagnostic efficiency of ACTL6A, serum CEA, and serum CAI9-9. *p<0.05.

Abbreviations: ACTL6A, actin like 6A; PC, pancreatic cancer; ROC, receiver operating characteristic curves; CAI9-9, carbohydrate antigen 19-9; CEA, carcinoembryonic antigen. 
Table I The Number of Patients in Different Scores Based on Immunohistochemistry Staining

\begin{tabular}{|l|c|c|}
\hline \multirow{2}{*}{ Scores } & \multicolumn{2}{|c|}{ No. of Patients (\%) } \\
\cline { 2 - 3 } & Tumor & Normal \\
\hline 1 & $11(18.3)$ & $17(28.3)$ \\
2 & $7(11.7)$ & $21(35.0)$ \\
3 & $1(1.7)$ & $6(10.0)$ \\
4 & $7(11.7)$ & $8(13.3)$ \\
6 & $24(40.0)$ & $8(13.3)$ \\
8 & $5(8.3)$ & $0(0.0)$ \\
9 & $2(3.3)$ & $0(0.0)$ \\
12 & $3(5.0)$ & $0(0.0)$ \\
\hline
\end{tabular}

\section{Diagnostic Value of ACTL6A Expression for PC}

To investigate the diagnostic value of ACTL6A expression for $\mathrm{PC}$, we performed ROC analysis on total scores of pancreatic cancer and normal pancreatic tissue, as shown in Figure 1D and E, and the AUC value was 0.724, which was higher than that of carbohydrate antigen 19-9 (CA19-9) and carcinoembryonic antigen (CEA). These results represented a moderate diagnostic value for PC. The specificity and sensitivity of ACTL6A expression for PC diagnosis were 0.867 and 0.567 , respectively. The cut-off value established for ACTL6A expression for the diagnosis of PC was 5.

\section{Relationships Between ACTL6A Expression and Clinicopathological Variables}

To further understand the role of ACTL6A in PC, we analyzed the relationship between ACTL6A expression and the clinicopathological characteristics. Patients with PC were divided into ACTL6A low-expression group (score $0-5 ; \mathrm{n}=34$ ) and ACTL6A high-expression group (score 6-12; $\mathrm{n}=26$ ) with the cut-off value of score 5. The relationship between ACTL6A expression and clinicopathological factors of pancreatic cancer is summarized in Table 2. Lymphovascular space invasion (LVSI) of PC was significantly associated with ACTL6A expression, which was more likely to occur in the ACTL6A high group. LVSI was present in 55.9\% (19/34) of patients in the ACTL6A high group and 26.9\% (7/26) in ACTL6A low group.

\section{Relationships Between ACTL6A}

\section{Expression and Overall Survival in PC}

The survival data of 178 PC patients was obtained from TCGA dataset. Patients are split into two groups according
Table 2 Relationships Between the Expression Level of ACTL6A and the Clinicopathological Characteristics of PC Patients

\begin{tabular}{|c|c|c|c|c|}
\hline \multirow[t]{2}{*}{ Characteristics } & \multirow[t]{2}{*}{$\begin{array}{c}\text { No. of } \\
\text { Patients }\end{array}$} & \multicolumn{2}{|c|}{$\begin{array}{l}\text { Expression of } \\
\text { ACTL6A }\end{array}$} & \multirow[t]{2}{*}{ p value } \\
\hline & & High & Low & \\
\hline Age(years) & & & & 0.151 \\
\hline$<65$ & 26 & 12 & 14 & \\
\hline$\geq 65$ & 34 & 22 & 12 & \\
\hline Sex & & & & 0.889 \\
\hline Male & 34 & 15 & 19 & \\
\hline Female & 26 & II & 15 & \\
\hline Tumor site & & & & 0.651 \\
\hline Head & 32 & 19 & 13 & \\
\hline Other ${ }^{\mathrm{a}}$ & 28 & 15 & 13 & \\
\hline Grade & & & & 0.713 \\
\hline Well/moderately & 40 & 22 & 18 & \\
\hline Poorly/ & 20 & 12 & 8 & \\
\hline undifferentiated & & & & \\
\hline T stage & & & & 0.505 \\
\hline $\mathrm{TI}+\mathrm{T} 2$ & 26 & 16 & 10 & \\
\hline $\mathrm{T} 3+\mathrm{T} 4$ & 34 & 18 & 16 & \\
\hline N stage & & & & 0.930 \\
\hline No & 25 & 14 & 11 & \\
\hline $\mathrm{N} 1+\mathrm{N} 2$ & 35 & 20 & 15 & \\
\hline M stage & & & & 0.339 \\
\hline MO & 57 & 31 & 26 & \\
\hline MI & 3 & 3 & 0 & \\
\hline TNM stage & & & & 0.702 \\
\hline I & 13 & 7 & 6 & \\
\hline II-IV & 47 & 27 & 20 & \\
\hline LVSI & & & & 0.025 \\
\hline Yes & 26 & 19 & 7 & \\
\hline No & 34 & 15 & 19 & \\
\hline
\end{tabular}

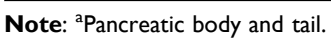

Abbreviations: ACTL6A, actin-Like 6A; PC, pancreatic cancer; LVSI, lymphovascular space invasion.

to the median value of Actl6a mRNA expression. One-half (89 patients) was defined as high Actl6a mRNA expression, and the other was defined as low Actl6a mRNA expression. Obviously, high Actl6a mRNA was associated with poor overall survival in patients with PC $(\mathrm{p}<0.001$, Figure 2A). Furthermore, based on data from our center, the Kaplan-Meier method was used to investigate the relationship between the expression of ACTL6A protein and OS of patients. The median OS in PC patients for the high and low expression of ACTL6A was $8.0 \pm 0.4$ months and $13.0 \pm 1.6$ months, respectively. Obviously, patients with low ACTL6A expression had significantly longer survival time than those with high ACTL6A expression $(\mathrm{p}<0.001$, Figure 2B). 

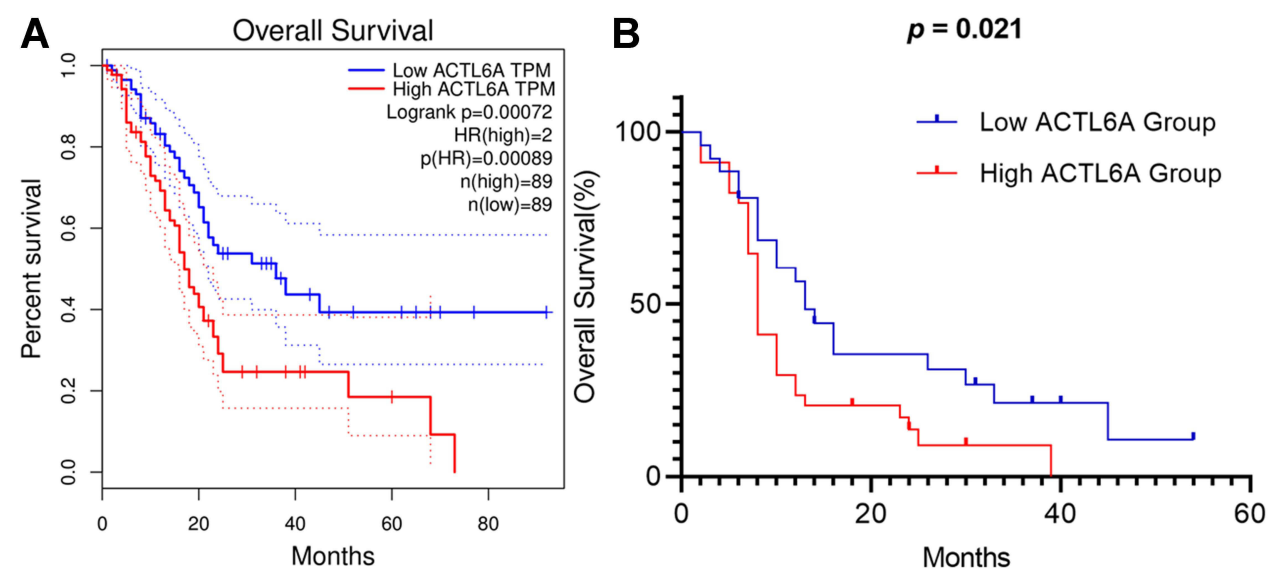

Figure 2 (A) Kaplan-Meier curves of overall survival in PC patients with high and low Actl6a mRNA expression. (B) Kaplan-Meier curves of overall survival in PC patients with high and low ACTL6A expression.

Abbreviations: ACTL6A, actin like 6A; PC, pancreatic cancer.

\section{ACTL6A Overexpression as an}

\section{Independent Risk Factor in Patients with PC}

Univariate and multivariate Cox analyses were performed to identify the prognostic factors on OS of patients with PC. The results demonstrated that ACTL6A overexpression $(p=0.032)$ and grade $(p=0.008)$ were risk factors for survival in patients with $\mathrm{PC}$ through univariate Cox analysis. Further multivariate Cox analysis showed that ACTL6A expression $(p=0.046)$ was an independent risk factor for poor prognosis of PC (Table 3). As shown in Figure $3 \mathrm{~A}$ and $\mathrm{B}$, the forest plot visualizes the specific $\mathrm{HR}$ of risk factors.

\section{Discussion}

Worldwide, PC has become a malignancy with a dismal prognosis and high mortality, which has a 5-year survival rate of less than $10 \%{ }^{19}$ There are two clinical features that are involved with the poor prognosis of PC. First, initial symptoms of $\mathrm{PC}$ are insidious, which leads to many challenges for early diagnosis. Second, PC has a significant potential for invasion and metastasis. ${ }^{20}$ In detail, the distant spread may occur in the early stages of PC, and more than $50 \%$ of patients with PC have no possibility to be treated with surgical resection. ${ }^{21}$ Scientific problems covering early diagnosis, the mechanisms of metastasis, and the risk factors of prognosis are necessary to be solved to improve survival of PC. In this study, we clarified that ACTL6A is highly expressed in PC, and it is a reliable marker for predicting the prognosis of PC patients.

ACTL6A is involved in a variety of cellular processes, including vesicle transport, spindle orientation, nuclear migration, and chromatin remodeling. ${ }^{7,22}$ Increasing evidence has suggested its involvement with tumorigenesis and development of cancer. ${ }^{7}$ ACTL6A has been reported to be overexpressed in a variety of malignancies, including hepatocellular carcinoma, ${ }^{9}$ ovarian cancer, ${ }^{18}$ cervical cancer, ${ }^{23}$ and esophageal squamous cell carcinoma, ${ }^{11}$ which is correlated with the prognosis of patients with malignancies. This evidence suggests that ACTL6A is a potential oncogene, and it is observed that ACTL6A expression is also upregulated in PC in our study, which is consistent with previous studies. Researchers have been constantly exploring diagnostic markers for PC. Jelski et al reported that the activity of alcohol dehydrogenase (ADH) class III isoenzyme in pancreatic cancer was significantly higher than that in normal tissues. ${ }^{24}$ And the total activity of $\mathrm{ADH}$ and class III isoenzyme was increased in the serum of patients with $\mathrm{PC}$, which can be due to the release of this isoenzyme from PC cells. ${ }^{25}$ Nevertheless, it was not observed that other types of ADH isoenzymes (I, II, IV) had a significant change in either pancreatic tissue or serum. Further exploration revealed that ADH III had the diagnostic value for $\mathrm{PC} .{ }^{26}$ Also, our evidence demonstrated a potential role for ACTL6A as a marker of PC.

ACTL6A plays a vital role in the invasion and metastasis of tumors by promoting EMT, leading to poor prognosis. ACTL6A expression is higher in fibroblasts and progenitor cells and inhibits the epithelial properties of epidermal tissues. ${ }^{27,28}$ Moreover, the functions of ACTL6A are similar to features of stem cells, including the inhibition of cell differentiation and the ability of self-renewal, which is closely related to the biological functions of EMT. ${ }^{28}$ In hepatocellular carcinoma, ACTL6A activated Notch1 signaling via 
Table 3 Univariate and Multivariate Analysis of Clinicopathological Characteristics Affecting Prognosis of Patients with PC

\begin{tabular}{|c|c|c|c|c|c|c|}
\hline \multirow[t]{2}{*}{ Characteristics } & \multicolumn{3}{|c|}{ Univariate } & \multicolumn{3}{|c|}{ Multivariate } \\
\hline & HR & $95 \% \mathrm{Cl}$ & $p$ value & HR & $95 \% \mathrm{Cl}$ & $p$ value \\
\hline \multicolumn{7}{|l|}{ ACTL6A } \\
\hline Low & & Ref & & & Ref & \\
\hline High & 1.899 & $1.056-3.415$ & 0.032 & 1.810 & $1.010-3.243$ & 0.046 \\
\hline \multicolumn{7}{|l|}{ Age(years) } \\
\hline$<65$ & & Ref & & & & \\
\hline$\geq 65$ & 1.053 & $0.600-1.848$ & 0.857 & & & \\
\hline \multicolumn{7}{|l|}{ Sex } \\
\hline Male & & Ref & & & & \\
\hline Female & 0.646 & $0.365-1.141$ & 0.132 & & & \\
\hline \multicolumn{7}{|l|}{ Tumor site } \\
\hline Head & & Ref & & & & \\
\hline Other $^{\mathrm{a}}$ & 0.736 & $0.422-1.284$ & 0.281 & & & \\
\hline \multicolumn{7}{|l|}{ Grade } \\
\hline Well/moderately & & Ref & & & Ref & \\
\hline Poorly/undifferentiated & 2.198 & $1.231-3.924$ & 0.008 & 2.021 & $1.127-3.624$ & 0.018 \\
\hline \multicolumn{7}{|l|}{ T stage } \\
\hline $\mathrm{TI}+\mathrm{T} 2$ & & Ref & & & Ref & \\
\hline $\mathrm{T} 3+\mathrm{T} 4$ & 1.737 & $0.973-3.103$ & 0.062 & 1.575 & $0.880-2.819$ & 0.126 \\
\hline \multicolumn{7}{|l|}{ N stage } \\
\hline No & & Ref & & & & \\
\hline $\mathrm{N} 1+\mathrm{N} 2$ & 1.442 & $0.816-2.550$ & 0.208 & & & \\
\hline \multicolumn{7}{|l|}{ M stage } \\
\hline Mo & & Ref & & & & \\
\hline MI & 2.205 & $0.673-7.221$ & 0.191 & & & \\
\hline \multicolumn{7}{|l|}{ TNM stage } \\
\hline 1 & & Ref & & & & \\
\hline II-IV & 1.759 & $0.853-3.626$ & 0.126 & & & \\
\hline \multicolumn{7}{|l|}{ LVSI } \\
\hline No & & Ref & & & & \\
\hline Yes & 1.434 & $0.822-2.501$ & 0.204 & & & \\
\hline
\end{tabular}

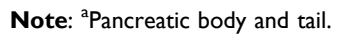

Abbreviations: ACTL6A, actin-Like 6A; PC, pancreatic cancer; LVSI, lymphovascular space invasion.

SOX2, which regulated EMT to affect the biological function and clinical prognosis of hepatocellular carcinoma. Other studies also revealed ACTL6A as an EMT activator to promote metastasis in osteosarcoma ${ }^{29}$ and colon cancer, ${ }^{10}$ respectively. Some studies mentioned the potential role of ACTL6A involvement with tumors. Zhang et al found that ACTL6A was a glycolytic regulator by phosphoglycerate kinase 1(PGK1) in ovarian cancer and participated in FSHinduced tumorigenesis of ovarian cancer. ${ }^{18}$ And in triple negative breast cancer, ACTL6A promoted tumor cell proliferation by enhancing the stability of MYC oncogene. ${ }^{30}$ Additional evidence suggested that ACTL6A promoted the progression of cervical cancer and laryngeal squamous cell carcinoma through activation of yes-associated protein (YAP) signaling. ${ }^{23,31}$ Besides, ACTL6A could stabilize transcriptional regulators YAP and transcriptional coactivator with PDZ-binding motif (TAZ) to regulate the proliferation, migration, and invasion of glioma. ${ }^{32}$ Further studies revealed that the knockdown of ACTL6A gene resulted in the inhibition of protein kinase $\mathrm{B}$ (AKT) signaling pathway to suppress cell migration and increased sensitivity of glioma cells to temozolomide. ${ }^{33}$ Moreover, in vivo and in vitro, Shrestha et al revealed that $\mathrm{p} 21^{\mathrm{Cip} 1}$, a tumor suppressor, was suppressed by ACTL6A in epidermal squamous cell carcinoma, leading to epidermal squamous cell carcinoma progression. ${ }^{34}$ More importantly, overexpressed ACTL6A was related to cisplatin-induced DNA damage and led to resistance to cisplatin. ${ }^{13}$ These studies have further confirmed the contribution of ACTL6A in the invasion, metastasis, and clinical prognosis of tumors.

In this research, we reveal a correlation between the expression of ACTL6A and the invasion and prognosis of 


\section{A Forest plot of univariate cox regression}

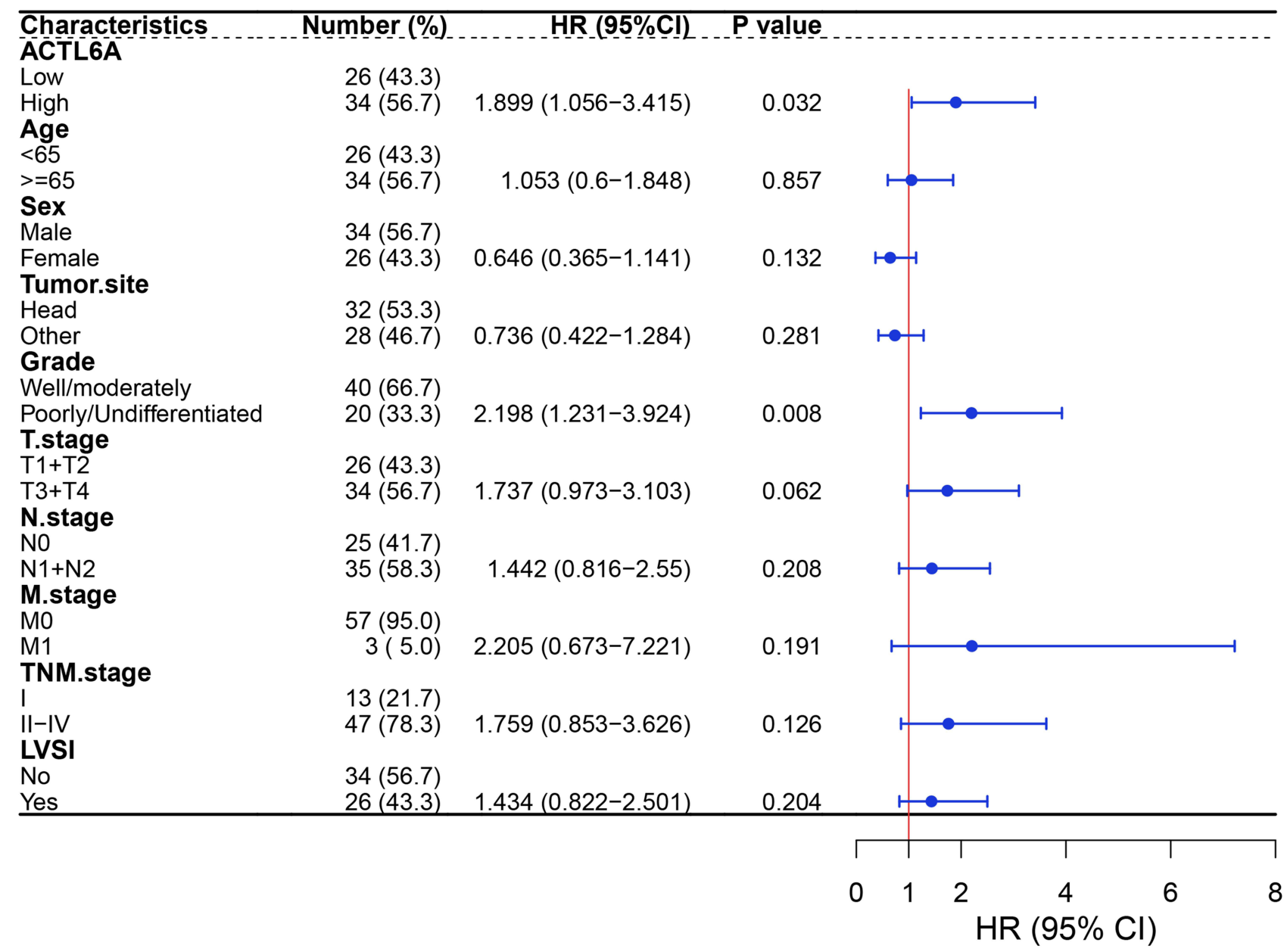

B $\quad$ Forest plot of multivariate cox regression

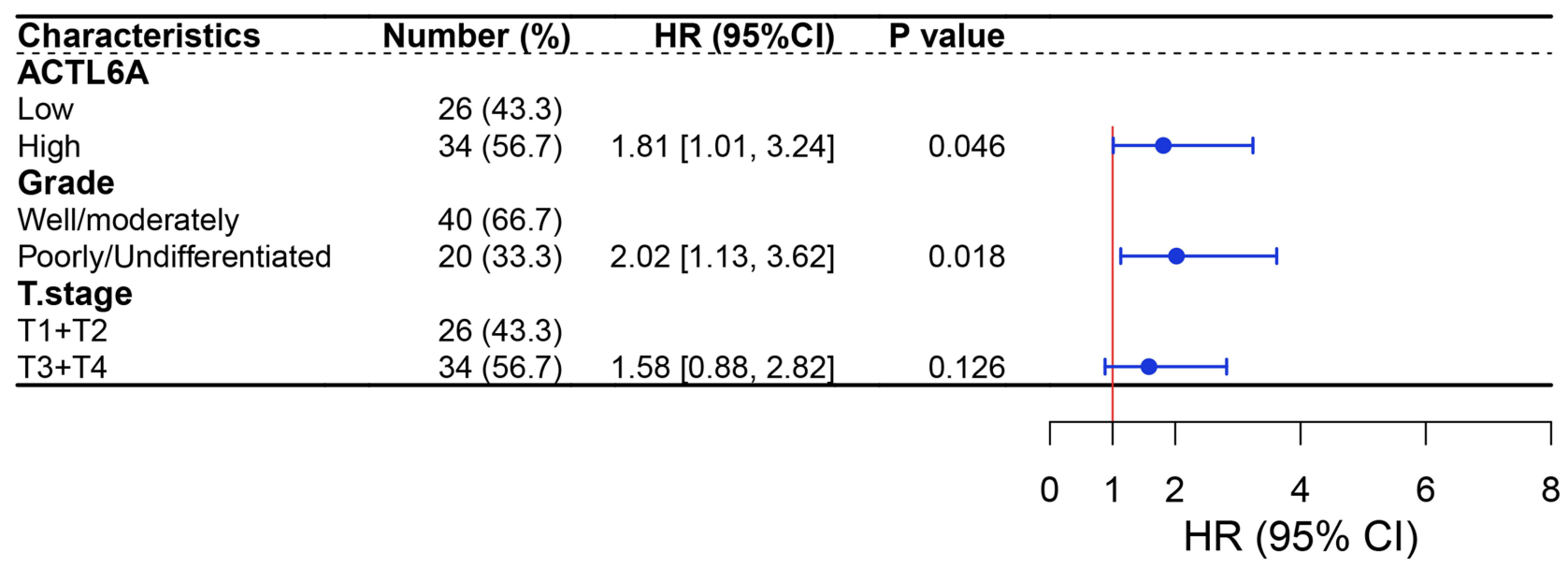

Figure 3 Forest plot of univariate $(\mathbf{A})$ and multivariate $(\mathbf{B})$ cox regression.

Abbreviations: ACTL6A, actin like 6A; PC, pancreatic cancer; LVSI, lymphovascular space invasion.

PC. It was found that LVSI was more likely to occur in PC patients with high ACTL6A expression, which might be related to the high aggressiveness caused by ACTL6A.
Univariate and multivariate Cox analysis suggested that ACTL6A expression and grade were independent risk factors for poor prognosis of PC. This study also 
confirmed ACTL6A as a valid prognostic biomarker and potential therapeutic target in PC. Given a follow-up and survival analysis of survival data of PC patients, patients with high ACTL6A expression had significantly poorer prognosis. It was suggested that ACTL6A expression in PC was a risk factor, which was consistent with the existing studies. And ACTL6A overexpression was associated with tumor progression. However, whether ACTL6A could induce PC cell proliferation, invasion, and metastasis in vitro, as well as the specific regulatory mechanisms, deserved further investigation.

\section{Conclusion}

In conclusion, it was found that levels of ACTL6A expression were elevated in PC tissues, which was associated with LVSI. Moreover, it was demonstrated that ACTL6A was an independent risk prognostic indicator for PC. ACTL6A could be used as a valuable biomarker to predict the prognosis of $\mathrm{PC}$, assisting clinicians to develop preventative measures and better treatment strategies to improve mortality in patients with PC.

\section{Acknowledgments}

The authors are grateful to all the patients, researchers and institutions that participated in the TCGA and GTEx database.

\section{Disclosure}

The authors report no conflicts of interest in this work.

\section{References}

1. Sung H, Ferlay J, Siegel RL, et al. Global cancer statistics 2020: GLOBOCAN estimates of incidence and mortality worldwide for 36 cancers in 185 countries. CA Cancer J Clin. 2021;71(3):209-249.

2. Zhang L, Sanagapalli S, Stoita A. Challenges in diagnosis of pancreatic cancer. World J Gastroenterol. 2018;24(19):2047-2060.

3. Mittal P, Roberts CWM. The SWI/SNF complex in cancer - biology, biomarkers and therapy. Nat Rev Clin Oncol. 2020;17(7):435-448.

4. Naito $T$, Udagawa $H$, Umemura $S$, et al. Non-small cell lung cancer with loss of expression of the SWI/SNF complex is associated with aggressive clinicopathological features, PD-L1-positive status, and high tumor mutation burden. Lung Cancer. 2019;138:35-42.

5. Cyrta J, Augspach A, De Filippo MR, et al. Role of specialized composition of SWI/SNF complexes in prostate cancer lineage plasticity. Nat Commun. 2020;11(1):5549.

6. Fukumoto T, Magno E, Zhang R. SWI/SNF complexes in ovarian cancer: mechanistic insights and therapeutic implications. Mol Cancer Res. 2018;16(12):1819-1825.

7. Krasteva V, Buscarlet M, Diaz-Tellez A, Bernard MA, Crabtree GR, Lessard JA. The BAF53a subunit of SWI/SNF-like BAF complexes is essential for hemopoietic stem cell function. Blood. 2012;120 (24):4720-4732.
8. Panwalkar P, Pratt D, Chung C, et al. SWI/SNF complex heterogeneity is related to polyphenotypic differentiation, prognosis, and immune response in rhabdoid tumors. Neuro Oncol. 2020;22 (6):785-796.

9. Xiao S, Chang RM, Yang MY, et al. Actin-like 6A predicts poor prognosis of hepatocellular carcinoma and promotes metastasis and epithelial-mesenchymal transition. Hepatology. 2016;63 (4):1256-1271.

10. Zeng Z, Yang H, Xiao S. ACTL6A expression promotes invasion, metastasis and epithelial mesenchymal transition of colon cancer. BMC Cancer. 2018;18(1):1020.

11. Li RZ, Li YY, Qin H, Li SS. ACTL6A promotes the proliferation of esophageal squamous cell carcinoma cells and correlates with poor clinical outcomes. Onco Targets Ther. 2021;14:199-211.

12. Nieto MA, Huang RY, Jackson RA, Thiery JP. Emt: 2016. Cell. 2016;166(1):21-45.

13. Xiao Y, Lin FT, Lin WC. ACTL6A promotes repair of cisplatin-induced DNA damage, a new mechanism of platinum resistance in cancer. Proc Natl Acad Sci U S A. 2021;118(3):e2015808118.

14. Cancer Genome Atlas Research N; Weinstein JN, Collisson EA, et al. The Cancer Genome Atlas Pan-Cancer analysis project. Nat Genet. 2013;45(10):1113-1120.

15. Consortium GT. Human genomics. The Genotype-Tissue Expression (GTEx) pilot analysis: multitissue gene regulation in humans. Science. 2015;348(6235):648-660.

16. Tang Z, Li C, Kang B, Gao G, Li C, Zhang Z. GEPIA: a web server for cancer and normal gene expression profiling and interactive analyses. Nucleic Acids Res. 2017;45(W1):W98-W102.

17. Rao X, Wang J, Song HM, Deng B, Li JG. KRT15 overexpression predicts poor prognosis in colorectal cancer. Neoplasma. 2020;67 (2):410-414.

18. Zhang J, Zhang J, Wei Y, Li Q, Wang Q. ACTL6A regulates follicle-stimulating hormone-driven glycolysis in ovarian cancer cells via PGK1. Cell Death Dis. 2019;10(11):811.

19. Zhu H, Li T, Du Y, Li M. Pancreatic cancer: challenges and opportunities. BMC Med. 2018;16(1):214.

20. Ansari D, Tingstedt B, Andersson B, et al. Pancreatic cancer: yesterday, today and tomorrow. Future Oncol. 2016;12(16):1929-1946.

21. Lamb YN, Scott LJ. Liposomal irinotecan: a review in metastatic pancreatic adenocarcinoma. Drugs. 2017;77(7):785-792.

22. Zhao K, Wang W, Rando OJ, et al. Rapid and phosphoinositol-dependent binding of the SWI/SNF-like BAF complex to chromatin after $\mathrm{T}$ lymphocyte receptor signaling. Cell. 1998;95(5):625-636.

23. Zhao J, Li L, Yang T. MiR-216a-3p suppresses the proliferation and invasion of cervical cancer through downregulation of ACTL6A-mediated YAP signaling. J Cell Physiol. 2020;235 (12):9718-9728.

24. Jelski W, Chrostek L, Szmitkowski M. The activity of class I, II, III, and IV of alcohol dehydrogenase isoenzymes and aldehyde dehydrogenase in pancreatic cancer. Pancreas. 2007;35(2):142-146.

25. Jelski W, Zalewski B, Szmitkowski M. Alcohol dehydrogenase $(\mathrm{ADH})$ isoenzymes and aldehyde dehydrogenase (ALDH) activity in the sera of patients with pancreatic cancer. Dig Dis Sci. 2008;53 (8):2276-2280.

26. Jelski W, Kutylowska E, Laniewska-Dunaj M, Szmitkowski M. Alcohol dehydrogenase (ADH) and aldehyde dehydrogenase (ALDH) as candidates for tumor markers in patients with pancreatic cancer. J Gastrointestin Liver Dis. 2011;20(3):255-259.

27. Bao X, Tang J, Lopez-Pajares V, et al. ACTL6a enforces the epidermal progenitor state by suppressing SWI/SNF-dependent induction of KLF4. Cell Stem Cell. 2013;12(2):193-203.

28. Lu W, Fang L, Ouyang B, et al. Actl6a protects embryonic stem cells from differentiating into primitive endoderm. Stem Cells. 2015;33 (6): 1782-1793. 
29. Sun W, Wang W, Lei J, Li H, Wu Y. Actin-like protein 6A is a novel prognostic indicator promoting invasion and metastasis in osteosarcoma. Oncol Rep. 2017;37(4):2405-2417.

30. Jian Y, Huang X, Fang L, et al. Actin-like protein $6 \mathrm{~A} / \mathrm{MYC} / \mathrm{CDK} 2$ axis confers high proliferative activity in triple-negative breast cancer. J Exp Clin Cancer Res. 2021;40(1):56.

31. Dang Y, Zhang L, Wang X. Actin-like 6A enhances the proliferative and invasive capacities of laryngeal squamous cell carcinoma by potentiating the activation of YAP signaling. J Bioenerg Biomembr. 2020;52(6):453-463.
32. Ji J, Xu R, Zhang X, et al. Actin like-6A promotes glioma progression through stabilization of transcriptional regulators YAP/TAZ. Cell Death Dis. 2018;9(5):517.

33. Chen X, Xiang Z, Li D, Zhu X, Peng X. ACTL6A knockdown inhibits cell migration by suppressing the AKT signaling pathway and enhances the sensitivity of glioma cells to temozolomide. Exp Ther Med. 2021;21(2):175.

34. Shrestha S, Adhikary G, Xu W, Kandasamy S, Eckert RL. ACTL6A suppresses p21(Cip1) expression to enhance the epidermal squamous cell carcinoma phenotype. Oncogene. 2020;39(36):5855-5866.

\section{Publish your work in this journal}

Cancer Management and Research is an international, peer-reviewed open access journal focusing on cancer research and the optimal use of preventative and integrated treatment interventions to achieve improved outcomes, enhanced survival and quality of life for the cancer patient.
The manuscript management system is completely online and includes a very quick and fair peer-review system, which is all easy to use. Visit http://www.dovepress.com/testimonials.php to read real quotes from published authors. 